\title{
Insights Into the Inhibition of MOX-1 $\beta$-Lactamase by S02030, a Boronic Acid Transition State Inhibitor
}

\author{
Tatsuya Ishikawa ${ }^{1 \dagger}$, Nayuta Furukawa ${ }^{2 \dagger}$, Emilia Caselli ${ }^{3}$, Fabio Prati ${ }^{3}$, \\ Magdalena A. Taracila ${ }^{4,5}$, Christopher R. Bethel ${ }^{4}$, Yoshikazu Ishii ${ }^{6}$, Akiko Shimizu-Ibuka ${ }^{1 *}$ \\ and Robert A. Bonomo $4,5,7,8 *$ \\ 1 Department of Applied Life Sciences, Niigata University of Pharmacy and Applied Life Sciences, Niigata, Japan, \\ ${ }^{2}$ Kagoshima Prefectural College, Kagoshima, Japan, ${ }^{3}$ Department of Life Sciences, University of Modena and Reggio \\ Emilia, Modena, Italy, ${ }^{4}$ Louis Stokes Cleveland Department of Veterans Affairs Medical Center, Cleveland, $\mathrm{OH}$, \\ United States, ${ }^{5}$ Department of Medicine, Case Western Reserve University School of Medicine, Cleveland, $\mathrm{OH}$, \\ United States, ${ }^{6}$ Department of Microbiology and Infectious Diseases, Toho University School of Medicine, Tokyo, Japan, \\ ${ }^{7}$ Departments of Pharmacology, Molecular Biology and Microbiology, Biochemistry, and Proteomics and Bioinformatics, \\ Case Western Reserve University School of Medicine, Cleveland, $\mathrm{OH}$, United States, ${ }^{8} \mathrm{CWRU}$-Cleveland VAMC Center \\ for Antimicrobial Resistance and Epidemiology (Case VA CARES), Cleveland, $\mathrm{OH}$, United States
}

The rise of multidrug resistant (MDR) Gram-negative bacteria has accelerated the development of novel inhibitors of class $A$ and $C \beta$-lactamases. Presently, the search for novel compounds with new mechanisms of action is a clinical and scientific priority. To this end, we determined the 2.13- $\AA$ resolution crystal structure of S02030, a boronic acid transition state inhibitor (BATSI), bound to MOX-1 $\beta$-lactamase, a plasmidborne, expanded-spectrum AmpC $\beta$-lactamase (ESAC) and compared this to the previously reported aztreonam (ATM)-bound MOX-1 structure. Superposition of these two complexes shows that S02030 binds in the active-site cavity more deeply than ATM. In contrast, the $\mathrm{SO}_{3}$ interactions and the positional change of the $\beta$-strand amino acids from Lys315 to Asn320 were more prominent in the ATM-bound structure. MICs were performed using a fixed concentration of S02030 $(4 \mu \mathrm{g} / \mathrm{ml})$ as a proof of principle. Microbiological evaluation against a laboratory strain of Escherichia coli expressing MOX-1 revealed that MICs against ceftazidime are reduced from 2.0 to $0.12 \mu \mathrm{g} / \mathrm{ml}$ when S02030 is added at a concentration of $4 \mu \mathrm{g} / \mathrm{ml}$. The $I_{50}$ and $K_{i}$ of S02030 vs. MOX-1 were $1.25 \pm 0.34$ and $0.56 \pm 0.03 \mu \mathrm{M}$, respectively. Monobactams such as ATM can serve as informative templates for design of mechanism-based inhibitors such as S02030 against ESAC $\beta$-lactamases.

Keywords: $\beta$-lactamase, BATSI, extended-spectrum AmpC, $\Omega$-loop, $\beta$-lactamase inhibitor

\section{INTRODUCTION}

The most widespread mechanism of resistance to $\beta$-lactam antibiotics among Gram-negative bacteria is the production of $\beta$-lactamases (EC 3.5.2.6) (Wilke et al., 2005). Based on their primary sequences, $\beta$-lactamases are grouped into four major classes, A-D (Ambler, 1980; Jaurin and Grundstrom, 1981; Ouellette et al., 1987). Class A, C, and D $\beta$-lactamases possess a conserved catalytic serine residue in their active site; class B harbors one or two zinc metal ions. Class $\mathrm{C}$ 
$\beta$-lactamases are widely distributed among many bacteria and show increased catalytic efficiency toward cephalosporins, and are poorly inhibited by the mechanism-based $\beta$-lactamase inhibitors (BLIs) clavulanic acid, sulbactam, and tazobactam (Bush et al., 1995; Helfand and Bonomo, 2003). New $\beta$-lactamase inhibitors recently developed are highly effective against a broader range of $\beta$-lactamases than clavulanic acid and tazobactam and have particular activity against class $C$ enzymes. These new inhibitors are from the diazabicyclooctanone (DBO) and boronate chemical families and both possess a $\beta$-lactamlike mode of action.

Boronic acid transition state analog inhibitors (BATSIs) are undergoing rapid development (Docquier and Mangani, 2018). S02030, a BATSI (Figure 1A), was found to inhibit a broad range of $\beta$-lactamases both in class $\mathrm{A}$ and class $\mathrm{C}$. Crystal structures of enzymes such as a class C $\beta$-lactamase ADC-7, class A enzymes SHV-1 and KPC-2 bound to S02030 have been reported (Powers et al., 2014; Nguyen et al., 2016). MOX-1 is a plasmid-mediated, CMY-type class C $\beta$-lactamase isolated from Klebsiella pneumoniae NU2936, a strain that is resistant to expanded-spectrum cephalosporins (Horii et al., 1993). Kinetic studies demonstrate that benzylpenicillin, cephalothin, cefcapene, and moxalactam are good substrates of MOX-1, while ceftazidime and cefepime are poor substrates and aztreonam (ATM; Figure 1C) behaves as an inhibitor (Alba et al., 2003).

The crystal structures of free and ATM-bound MOX-1 have been determined (Oguri et al., 2014, 2015). In both analyses, electron density for some amino acids in the $\Omega$-loop (Asn212Pro213) and N-terminus of B3 $\beta$-strand (from Glu306 to Ser309) were not observed, suggesting significant flexibility in these regions. The structure of ATM-bound MOX-1 (ATM: MOX-1) also revealed that the binding of ATM causes conformational change and structural disturbance of the B3 $\beta$-strand main chain (Oguri et al., 2015). In this present study, we determined the 2.13- $\AA$ resolution crystal structure of S02030 bound to MOX$1 \beta$-lactamase. Difference of binding mode between S02030 and ATM was analyzed, and the correlation between the binding mode and inhibitory activity was investigated. Our goal was to exploit similarities and differences in binding between ATM and S02030 in order to inform better inhibitor design. We also performed comparative analysis of the S02030 binding mode in several structure-solved complexes. Based on this analysis, four new derivatives of S02030 were proposed for further investigation. The binding pattern of the new designed inhibitors was determined using in silico studies, by docking them into the active site of MOX-1 enzyme. In order to facilitate comparison, the amino acid residues of MOX-1 are designated with the standard numbering for class C $\beta$-lactamases as previously reported (Mack et al., 2020).

\section{MATERIALS AND METHODS}

\section{Preparation of MOX-1}

Production and purification of MOX-1 with (His) $)_{8}$ tag at its $\mathrm{N}$-terminus were performed as described previously (Oguri et al., 2014). For kinetic assays, the purified protein was treated with thrombin to remove the $(\mathrm{His})_{8}$ tag. The sample was further purified with Hi-Trap Benzamidine FF column and His-Trap FF column (Cytiva).

\section{Kinetic Analysis}

S02030 was chemically synthesized as previously described (Powers et al., 2014). Cephalothin (TIN) $\left(\Delta \varepsilon_{262}=-7,660 \mathrm{M}^{-1} \mathrm{~cm}^{-1}\right)$ was purchased from SigmaAldrich and used as substrate. ATM was purchased from Tokyo Chemical Industry Co., Ltd., MOX-1 was pre-incubated with inhibitor (S02030 or ATM) in assay buffer for $10 \mathrm{~min}$ before initiation of the reaction. The reaction was performed in $50 \mathrm{mM}$ MOPS buffer ( $\mathrm{pH} 7.0$ ) containing $200 \mathrm{mM} \mathrm{NaCl}$ at $30^{\circ} \mathrm{C}$ with Jasco V-530 UV/VIS spectrophotometer. $K_{\mathrm{i}}$ and $\mathrm{IC}_{50}$ were determined as previously described (Drawz et al., 2011; Caselli et al., 2015).

\section{Minimal Inhibitory Concentration Assays}

Escherichia coli BL21(DE3) cell (New England Biolabs) transformed with pET-28a plasmid harboring the MOX-1 gene was used for susceptibility assays. Minimal inhibitory concentration (MIC) assays were performed by a broth microdilution method following the guidelines of the Clinical and Laboratory Standards Institutes (CLSI, 2012). An inoculum of $10^{4} \mathrm{E}$. coli cells was grown in Mueller-Hinton broth at $37^{\circ} \mathrm{C}$ for $20 \mathrm{~h}$ in the presence of ATM with or without S02030 $(4 \mu \mathrm{g} / \mathrm{ml})$, supplied in serial dilutions in a 96-well microplate. E. coli BL21(DE3) cells transformed with the pET-28a plasmid without the MOX-1 gene served as negative control.

\section{Crystal Preparation, Data Collection, and Structure Determination}

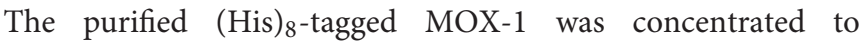
$10 \mathrm{mg} / \mathrm{mL}$ with Amicon Ultra-4 $10 \mathrm{~K}$ (Merck Millipore Ltd.), and it was subjected to crystallization. Crystals for X-ray analysis were grown at $16^{\circ} \mathrm{C}$ by the hanging-drop vapor diffusion method with reservoir solutions of $14-20 \%$ polyethylene glycol 8,000 in $100 \mathrm{mM}$ sodium cacodylate buffer ( $\mathrm{pH}$ 6.5) with $0.2 \mathrm{M}$ zinc acetate. Crystals were soaked with S02030 by dipping in a reservoir solution containing 5-10 mM S02030 for 10$60 \mathrm{~min}$. These crystals were flash frozen in a liquid nitrogen stream $\left(100^{\circ} \mathrm{K}\right)$ with $20 \%$ ethylene glycol as a cryoprotectant and immediately subjected to data collection at Station 5A of the Photon Factory, the High Energy Accelerator Research Organization (KEK, Ibaraki, Japan). The reflection was indexed and integrated using iMosflm and SCALA in CCP4 package (Collaborative Computational Project Number 4, 1994). The initial model for molecular replacement was the structure of free MOX-1 (PDB entry: 3W8K). Molecular replacement and refinement were performed with MOLREP in CCP4 (Vagin and Teplyakov, 2010). The coordinate and structure factor files have been deposited in the Protein Data Bank under the accession code $5 Z \mathrm{Z}$ B.

\section{Molecular Docking}

The crystal structure of MOX-1 (PDB: $3 \mathrm{~W} 8 \mathrm{~K}$ ) was used to create the models. The structure was minimized using Discovery Studio (BIOVIA, 2020) molecular modeling software (BIOVIA, 2020). 

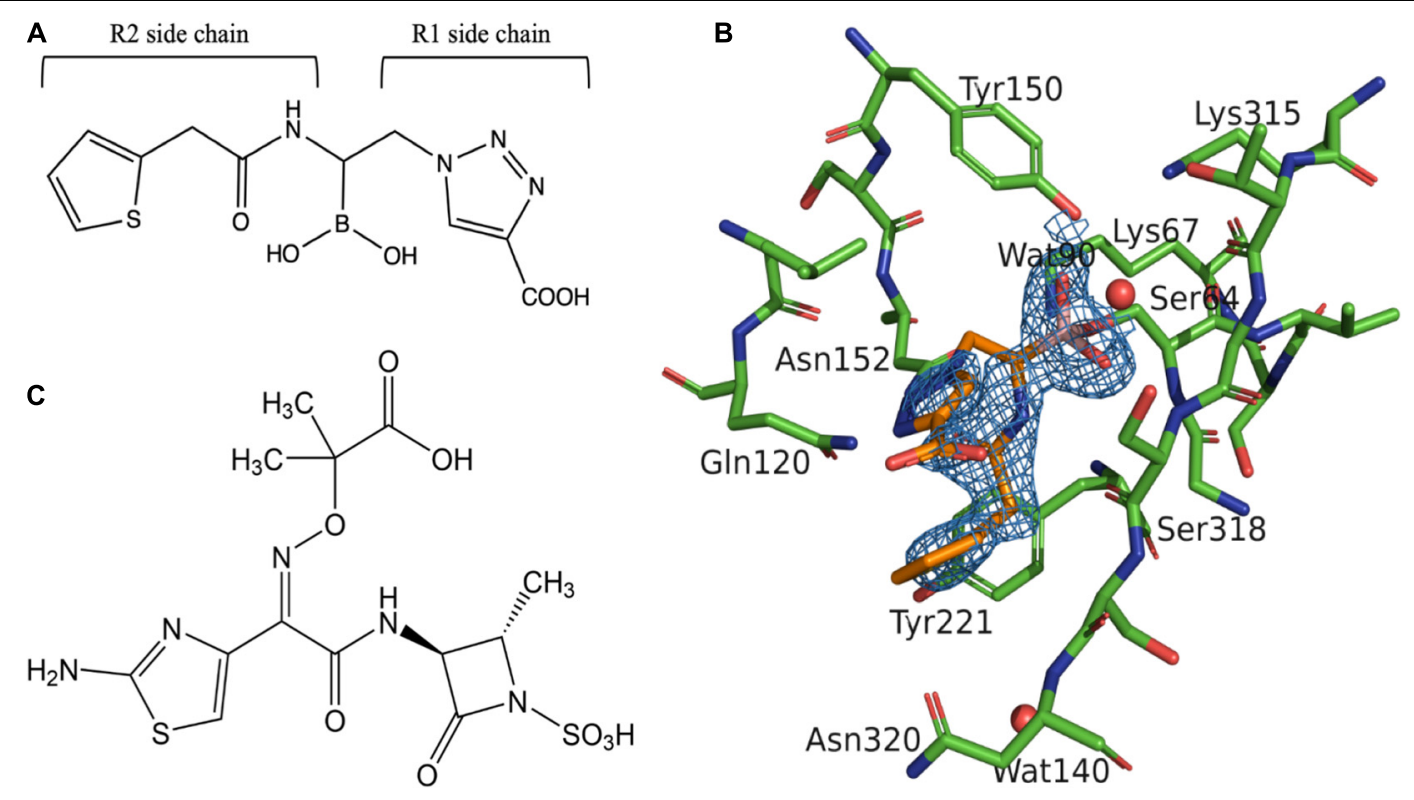

FIGURE 1 | Structure of aztreonam (ATM) and S02030 bound to MOX-1 $\beta$-lactamase. (A) Chemical structure of S02030. (B) Electron density of S02030 bound to the active site of MOX-1. (C) Chemical structure of ATM.

The water molecules were deleted, and the new compounds were built and minimized. CDOCKER (Wu et al., 2003) protocol was used to dock the new compounds into the active site of MOX1 and analyzed.

\section{RESULTS AND DISCUSSION}

\section{Inhibition Kinetics and Microbiological Data (MIC)}

In order to assess the affinity of the studied ligands, we determined both the $K_{\mathrm{i}}$ and $\mathrm{IC}_{50}$. S02030 and ATM were incubated with MOX-1 for $10 \mathrm{~min}$ and velocities were determined. The $\mathrm{IC}_{50}$ and $K_{\mathrm{i}}$ values for S02030 were $1.25 \pm 0.34 \mu \mathrm{M}$ and $0.563 \pm 0.030 \mu \mathrm{M}$, respectively. These values are greater than those for ATM (Table 1) and suggest that ATM is a more potent inhibitor with higher affinity to MOX-1 enzyme than S02030.

To assess the microbiological potency of S02030 compared to ATM, we determined MICs in a uniform genetic background (Table 2). Against E. coli BL21 (DE3) expressing MOX-1, the MICs of ATM are $1 \mu \mathrm{g} / \mathrm{ml}$. With $4 \mu \mathrm{g} / \mathrm{ml}$ of S02030, MICs were reduced to $0.125 \mu \mathrm{g} / \mathrm{ml}$. With regards to cefoxitin (FOX), MICs were reduced from 32 to $8 \mu \mathrm{g} / \mathrm{ml}$ using the same concentration

TABLE 1 | IC50 and Ki values of S02030 and aztreonam.

\begin{tabular}{lcc}
\hline Inhibitor & $\mathbf{I C}_{\mathbf{5 0}}(\boldsymbol{\mu} \mathbf{M})$ & $\boldsymbol{K}_{\boldsymbol{i}}(\boldsymbol{\mu} \mathbf{M})$ \\
\hline S02030 & $1.25 \pm 0.34$ & $0.56 \pm 0.03$ \\
Aztreonam & $0.02 \pm 0.01$ & $0.010 \pm 0.001$
\end{tabular}

of S02030. For ceftazidime (CAZ), MICs were reduced by four dilutions, from 2 to $0.125 \mu \mathrm{g} / \mathrm{ml}$.

\section{Crystal Structure}

The crystal structure of S02030-bound MOX-1 (S02030: MOX1) was determined at 2.13- $\AA$ resolution with $R$ and $R_{\text {free }}$ factors of 15.8 and $20.7 \%$, respectively (Table 3 ). Space group and cell parameters are basically identical with the previous data for free and ATM-bound MOX-1, and there is one molecule in an asymmetric unit.

The final S02030: MOX-1 model includes amino acid residues from Asp6 to Arg305 and from Gln310 to Gly362. The overall structure is almost identical to that of free MOX-1 (PDB accession code: $3 \mathrm{~W} 8 \mathrm{~K}$ ), with a root-mean-square deviation (RMSD) of $0.099 \AA$ for all atoms. Electron density for the $\mathrm{N}$-terminus of B3 $\beta$-strand (from Glu306 to Ser309) was not observed as in the case of previous structural analyses of MOX1. On the other hand, Asn213-Pro214, a part of $\Omega$ loop, which was unclear in the previous structural analyses of free and ATMbound MOX-1, is observed in this S02030-bound structure.

\section{Binding Mode of S02030}

In S02030: MOX-1 structure, the temperature factor for S02030 is high, and the electron density for solvent exposed tail of R2 side chain is not clearly observed (Table 3 and Figure 1B). The S02030 boron atom is covalently attached to the catalytic Ser64 residue, and there are several hydrogen bonds between the inhibitor and the enzyme (Figure 2A). The oxygen atom of the borate hydroxyl group is positioned in the oxyanion hole and makes hydrogen bonds with the main chain nitrogen and oxygen atoms of Ser318, and nitrogen atom of Ser64. The oxygen atom of the other hydroxyl group is hydrogen-bonded to the side-chain 
TABLE 2 | MIC data of antibiotics* in presence and absence of S02030 (4 $\mu \mathrm{g} / \mathrm{ml})$.

\begin{tabular}{|c|c|c|c|c|c|c|}
\hline & ATM & ATM + S02030 & FOX & $F O X+S 02030$ & CAZ & $\mathrm{CAZ}+\mathrm{S} 02030$ \\
\hline $\begin{array}{l}\text { E. coli BL21(DE3) } \\
\text { bla }_{\mathrm{MOX}-1} / \mathrm{pET}-28 \mathrm{a}\end{array}$ & 1 & 0.125 & 32 & 8 & 2 & 0.12 \\
\hline
\end{tabular}

${ }^{\star}$ Aztreonam (ATM), cefoxitin (FOX), and ceftazidime (CAZ).

oxygen atom of Tyr150. The nitrogen atom of the R1 amide group interacts with the main chain oxygen atom of Ser318, and the oxygen of the same group interacts with the side chain nitrogen atoms of Gln120 and Asn152.

\section{Structural Comparison Between S02030 and Aztreonam Bound to MOX-1}

The structure of MOX-1 in S02030-bound MOX-1 (S02030: MOX-1) is similar to that in ATM-bound MOX-1 (ATM: MOX1 , and PDB entry code $4 \mathrm{WBG}$ ), with a RMSD or $0.113 \AA$ for all atoms. Comparison of hydrogen bonding pattern in these two complexes suggests that ATM is more tightly bound to the active site of the enzyme through its $\mathrm{SO}_{3}$ group, since several hydrogen bonds are formed between this group and the active-site residues of MOX-1 (Figure 2B), and electron density for this $\mathrm{SO}_{3}$ group was fairly high (Oguri et al., 2015). Structural superposition of

TABLE 3 | Data collection and refinement statistics.

\begin{tabular}{|c|c|}
\hline \multicolumn{2}{|l|}{ Data collection } \\
\hline Space group & $P 2_{1}$ \\
\hline Cell parameters $(\AA$, deg $)$ & $\begin{array}{c}a=49.64, b=59.20, c=62.89 \\
\alpha=\gamma=90.00, \beta=102.03\end{array}$ \\
\hline Resolution $(\AA)$ & $2.13-61.51(2.13-2.25)$ \\
\hline Observed reflections & $63550(9337)$ \\
\hline Unique reflections & $20024(2908)$ \\
\hline Completeness (\%) & 99.9 (100.0) \\
\hline Redundancy & $3.2(3.2)$ \\
\hline$R_{\text {merge }}$ & $0.079(0.188)$ \\
\hline$/ / \sigma(I)$ & $10.8(5.5)$ \\
\hline \multicolumn{2}{|l|}{ Refinement } \\
\hline Resolution range for refinement $(\AA)$ & $2.13-42.69(2.13-2.18)$ \\
\hline Unique reflections & $19050(1397)$ \\
\hline Completeness (\%) & $99.84(100.00)$ \\
\hline Number of atoms & 2881 \\
\hline Protein & 2631 \\
\hline Water & 190 \\
\hline Other atoms & 60 \\
\hline$R_{\text {work }}$ & $0.158(0.173)$ \\
\hline$R_{\text {free }}$ & $0.205(0.231)$ \\
\hline \multicolumn{2}{|l|}{ Average B factor $\left(\AA^{2}\right)$} \\
\hline All atoms & 24.88 \\
\hline Protein & 24.13 \\
\hline Ligand (ZXM) & 56.31 \\
\hline Water & 29.82 \\
\hline Other solvent molecules & 24.54 \\
\hline \multicolumn{2}{|l|}{ Root mean square deviation } \\
\hline Bond lengths $(\AA)$ & 0.009 \\
\hline Bond angles (deg) & 1.279 \\
\hline
\end{tabular}

these two structures indicates that S02030 binds to the activesite cavity more deeply than ATM (Figure 2C). Binding of ATM effected a conformational change and/or structural disturbance in the $\beta$-strand amino acids from Lys315 to Asn320, with significantly high $\mathrm{B}$-factor values in their $\mathrm{C} \alpha$ atoms (Oguri et al., 2015). The binding of S02030 does not impact the position of these residues, with no elevation of B-factor values in this region.

\section{Comparison of S02030 Binding With Other $\beta$-Lactamases}

The binding position of S02030 to the active-site cavity of MOX1 was compared with that of S02030-bound class C $\beta$-lactamase ADC-7 (PDB entry code: 4U0X) (Figure 3A; Powers et al., 2014). Four independent molecules determined in the crystallographic analysis of S02030-bound ADC-7 showed some variety in the position of S02030, and here we compared our S02030: MOX-1 structure with the $\mathrm{C}$ monomer.

The binding mode is very similar in both enzymes, except the position of R2 side chain. In the S02030: ADC-7 complex, the carboxylate group of R2 group interacts with Arg343. The position of this Arg is occupied with Asn in MOX-1, and lack of the interaction should cause the difference in the position and flexibility of R2 side chain between these two enzymes. This is in agreement with the fact that the electron density for the R2 side chain is less clear in S02030: MOX-1 than S02030: ADC-7, and with the $K_{\mathrm{i}}$ values of $\mathrm{S} 02030$ for these two enzymes: $K_{\mathrm{i}}$ for MOX-1 was $563 \pm 30 \mathrm{nM}$, while that for ADC-7 was reported to be $44.5 \mathrm{nM}$. It was also reported that SM23, an inhibitor with shorter R2 group which allow to form more favorable interaction with Arg343, has higher affinity for ADC-7 than S02030 (Powers et al., 2014).

S02030 binds to the active site in a shallower position in class $\mathrm{A}$ $\beta$-lactamase KPC-2 (PDB entry code: 5EEC) (Figure 3B). This is due to the structural difference that class A KPC-2 has its $\Omega$ loop of helical structure that narrows the binding site for R1 group. Binding of boron oxygen with Glu166 and Asn170 in class A enzymes also changes the orientation of amide chain of S02030 R1 group (Nguyen et al., 2016).

To take advantage of the possible interaction of inhibitors with Lys315, as seen with the ATM interaction and Arg346 (present in both ADC-7 and MOX-1), and shorter side chain of Asn343 vs. Arg343 in ADC-7, the carboxyl group of S02030 was replaced by a series of negatively charged groups (Figure 4A). The new compounds with sulfate group $\mathrm{SO}_{3}(\mathrm{a})$, tetrazole (b), phosphate (c), and double carboxyl (d) were docked into the active site of MOX-1; the results show that the $\mathrm{SO}_{3}$ group (Figure 4B) makes interactions with Lys315 and Arg346. Interestingly, the double carboxyl group additionally forms interactions not only with Asn343, Arg346, but also with Lys205 (Gln205 in ADC-7). 

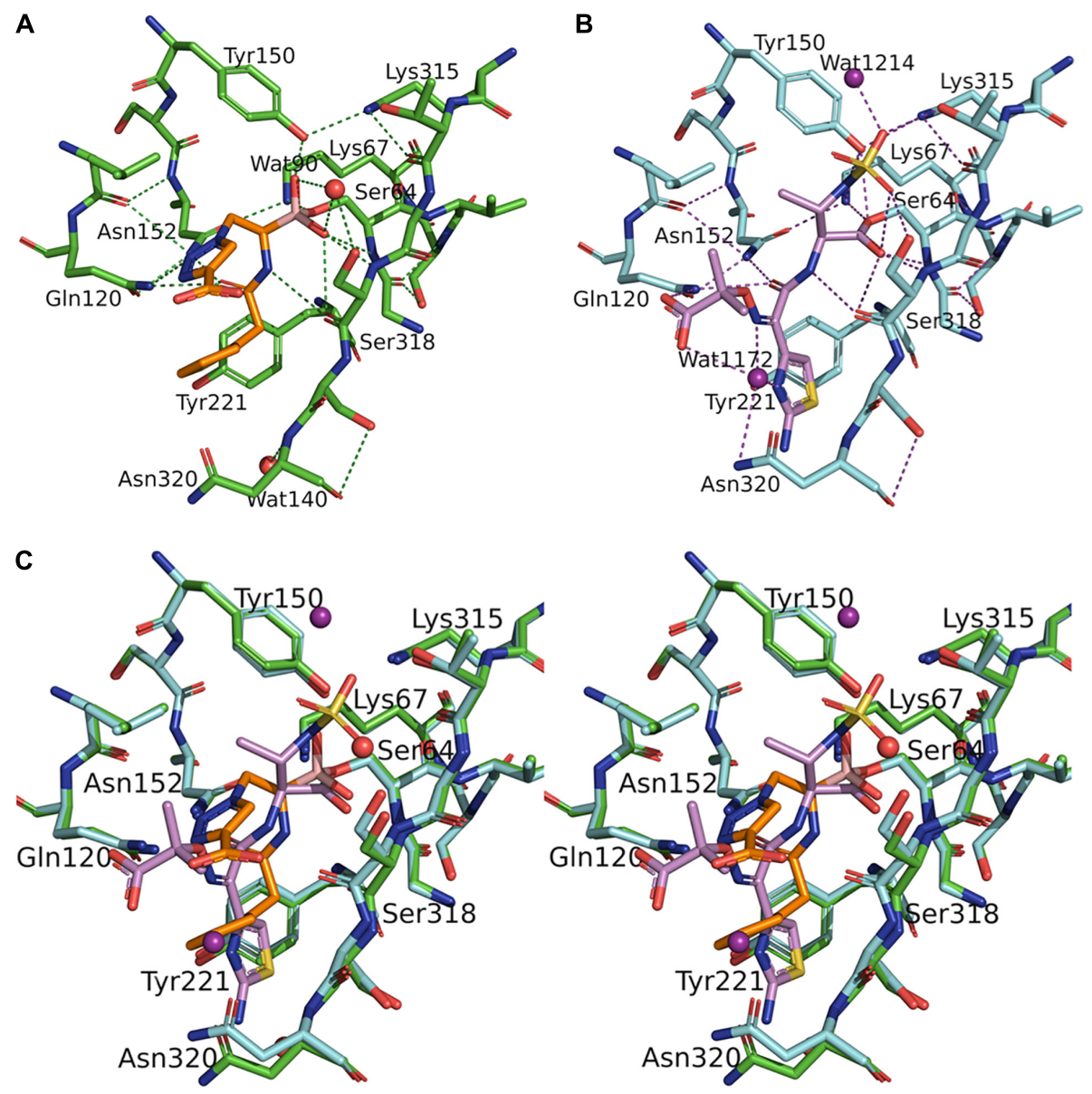

FIGURE 2 | Structural comparison between S02030: MOX-1 and ATM: MOX-1. Hydrogen-bonding pattern between S02030 and MOX-1 (A) and between ATM and MOX-1 (B). (C) Superposition of active site structures of S02030: MOX-1 (orange: green) and ATM: MOX-1 (violet: cyan).
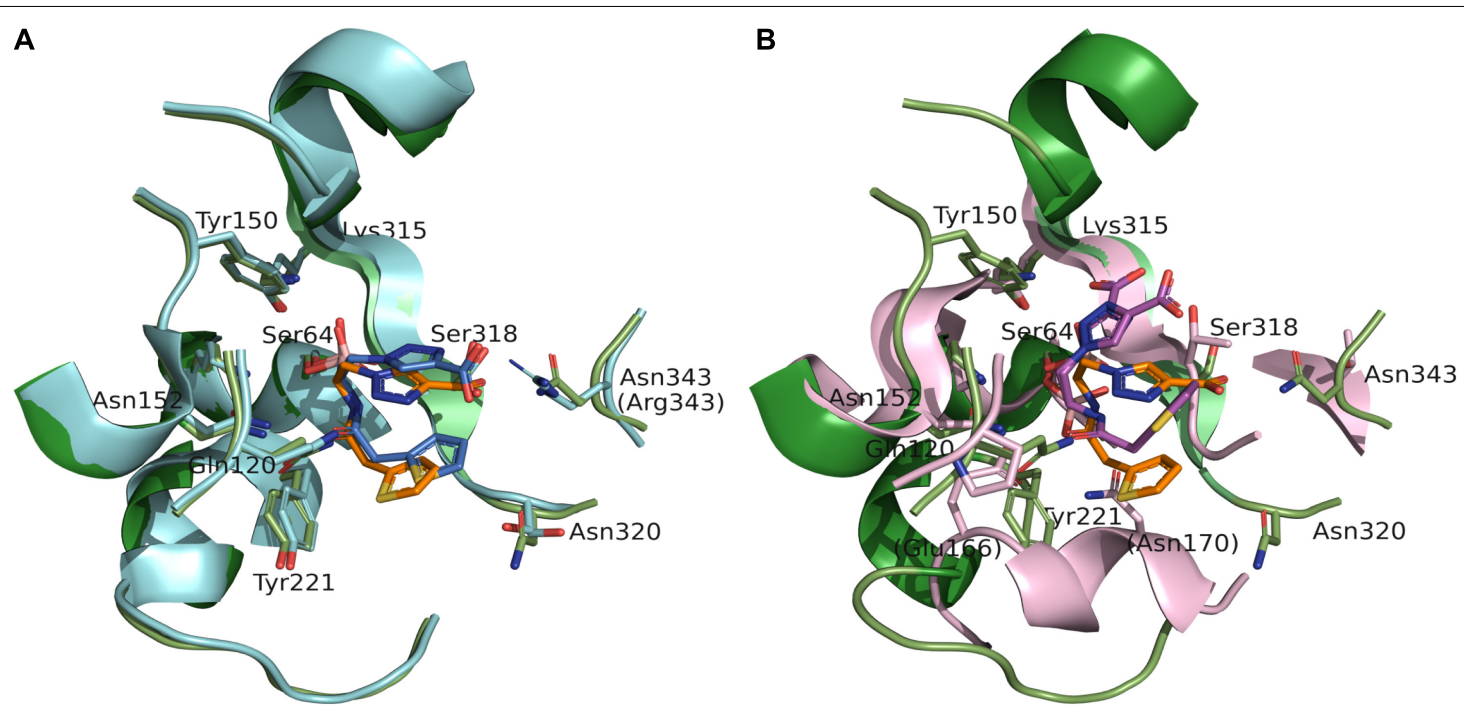

FIGURE 3 | Superposition of S02030: MOX-1 (orange: green) and previously analyzed crystal structures of S02030: ADC-7 (PDB: 4U0X) [(A) blue: cyan] and S02030: KPC-2 (PDB: 5EEC) [(B) violet: pink]. The labels in parentheses indicate the residues of ADC-7 (A) and KPC-2 (B) 
A

S02030

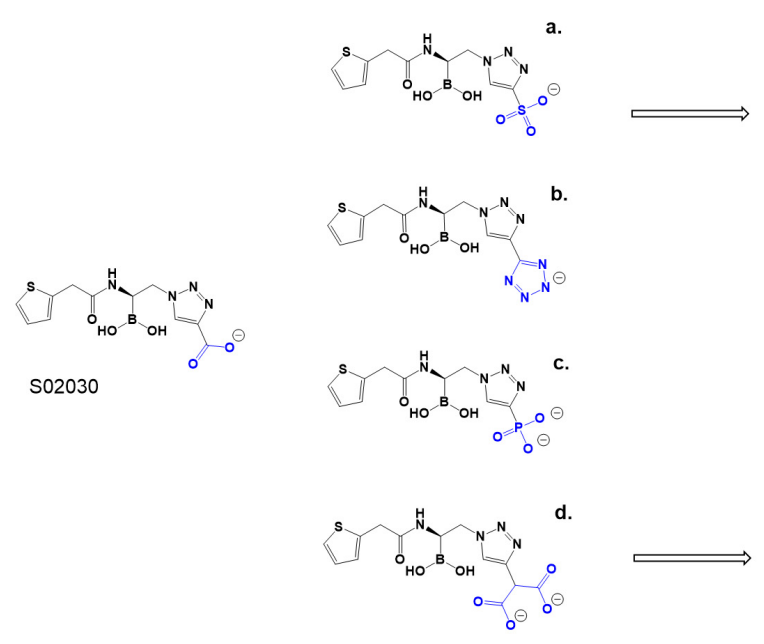

B

B

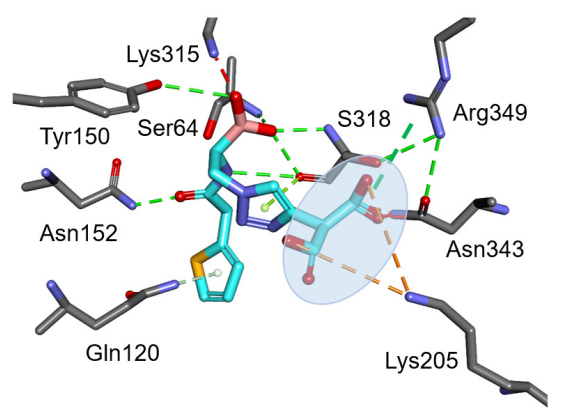

FIGURE 4 | Proposed derivatives of S02030 that mimic ATM (A) docked as acyl enzyme complexes into the active site of MOX-1 (B). The new added moieties are targeting the active site residues Lys315, Asn343, and new interactions with Arg349 and Lys205 for better inhibition.

The interactions with Gln120 and Asn152 were preserved, as well as the interactions of boronic groups with Tyr150 and Ser318.

\section{CONCLUSION}

Inhibition of extended-spectrum $\beta$-lactamases is a continuing challenge. In class $\mathrm{C} \beta$-lactamase MOX-1, the $\mathrm{B} 3 \beta$-strand amino acids from Lys315 to Asn320 change position when complexed with ATM in contrast to S02030, a BATSI effective both for class A and class C enzymes. S02030, as a transition state mimic, binds more deeply in the active site and does not impact a conformational change. Hydrogen-bonding interaction between inhibitors and MOX-1 shows that ATM is more tightly bound to the active site through its $\mathrm{SO}_{3}$ group, a feature missing in $\mathrm{S} 02030$. Such structural features are consistent with the result of kinetic analysis that showed that the $\mathrm{IC}_{50}$ and $K_{\mathrm{i}}$ values for S02030 are greater than those for ATM, indicating that S02030 behaves as a less potent inhibitor than ATM. These observations suggest that a novel approach forward in BATSI design would be to add an $\mathrm{SO}_{3}$ group in the region of the R1 side chain to effect greater hydrogen binding opportunities.

\section{DATA AVAILABILITY STATEMENT}

The datasets presented in this study can be found in online repositories. The names of the repository/repositories and accession number(s) can be found in the article/ supplementary material.

\section{AUTHOR CONTRIBUTIONS}

TI and NF performed the kinetic and X-ray crystallographic analysis. EC and FP designed and synthesized S02030. MT performed the MICs and molecular modeling. RAB conceived of the experiment, directed research, and contributed to wrote the manuscript. YI performed the MIC investigation and validation, and contributed to writing the manuscript. AS-I and CB contributed to writing the manuscript. All authors contributed to the article and approved the submitted version.

\section{FUNDING}

Research reported in this publication was supported by the National Institute of Allergy and Infectious Diseases of the National Institutes of Health (NIH) to RAB under Award Numbers R01AI100560, R01AI063517, and R01AI072219. This study was also supported in part by funds and/or facilities provided by the Cleveland Department of Veterans Affairs, Award Number 1I01BX001974 to RAB from the Biomedical Laboratory Research and Development Service of the VA Office of Research and Development, and the Geriatric Research Education and Clinical Center VISN 10.

\section{ACKNOWLEDGMENTS}

We thank Masahiro Nakajima and KEK staff members for their kind support and advice during data collection at KEK. 


\section{REFERENCES}

Alba, J., Bauvois, C., Ishii, Y., Galleni, M., Masuda, K., Ishiguro, M., et al. (2003). A detailed kinetic study of Mox-1, a plasmid-encoded class C $\beta$-lactamase. FEMS Microbiol. Lett. 225, 183-188. doi: 10.1016/S0378-1097(03)00448-8

Ambler, R. P. (1980). The structure of $\beta$-lactamases. Philos. Trans. R. Soc. Lond. $B$ Biol. Sci. 289, 321-331.

BIOVIA (2020). Discovery Studio 2020 Client, 3DEXPERIENCER Platform. San Diego, CA: Dassault Systèmes. Available online at: https://www.3ds.com/biovia

Bush, K., Jacoby, G. A., and Medeiros, A. A. (1995). A functional classification scheme for $\beta$-lactamases and its correlation with molecular structure. Antimicrob. Agents Chemother. 39, 1211-1233. doi: 10.1128/AAC.39.6. 1211

Caselli, E., Romagnoli, C., Vahabi, R., Taracila, M. A., Bonomo, R. A., and Prati, F. (2015). Click chemistry in lead optimization of boronic acids as beta-lactamase inhibitors. J. Med. Chem. 58, 5445-5458. doi: 10.1021/acs.jmedchem.5b00341

CLSI (2012). Methods for Dilution Antimicrobial Susceptibility Tests for Bacteria That Grow Aerobically; Approved Standard, 9th Edn. Wayne, PA: Clinical and Laboratory Standards Institute.

Collaborative Computational Project Number 4 (1994). The CCP4 suite: programs for protein crystallography. Acta Crystallogr. D Biol. Crystallogr. 50, 760-763.

Docquier, J. D., and Mangani, S. (2018). An update on beta-lactamase inhibitor discovery and development. Drug. Resist. Updat. 36, 13-29.

Drawz, S. M., Taracila, M., Caselli, E., Prati, F., and Bonomo, R. A. (2011). Exploring sequence requirements for $\mathrm{C}(3) / \mathrm{C}(4)$ carboxylate recognition in the Pseudomonas aeruginosa cephalosporinase: insights into plasticity of the AmpC beta-lactamase. Protein Sci. 20, 941-958. doi: 10.1002/pro.612

Helfand, M. S., and Bonomo, R. A. (2003). Beta-lactamases: a survey of protein diversity. Curr. Drug Targets Infect. Disord. 3, 9-23.

Horii, T., Arakawa, Y., Ohta, M., Ichiyama, S., Wacharotayankun, R., and Kato, N. (1993). Plasmid-mediated AmpC-type beta-lactamase isolated from Klebsiella pneumoniae confers resistance to broad-spectrum beta-lactams, including moxalactam. Antimicrob. Agents Chemother. 37, 984-990. doi: 10.1128/AAC. 37.5.984

Jaurin, B., and Grundstrom, T. (1981). ampC cephalosporinase of Escherichia coli K-12 has a different evolutionary origin from that of beta-lactamases of the penicillinase type. Proc. Natl. Acad. Sci. U.S.A. 78, 4897-4901. doi: 10.1073/ pnas.78.8.4897

Mack, A. R., Barnes, M. D., Taracila, M. A., Hujer, A. M., Hujer, K. M., Cabot, G., et al. (2020). A standard numbering scheme for Class C beta-Lactamases. Antimicrob. Agents Chemother. 64:e02247-19. doi: 10.1128/AAC.01841-19

Nguyen, N. Q., Krishnan, N. P., Rojas, L. J., Prati, F., Caselli, E., Romagnoli, C., et al. (2016). Crystal structures of KPC-2 and SHV-1 beta-Lactamases in complex with the boronic acid transition state analog S02030. Antimicrob. Agents Chemother. 60, 1760-1766. doi: 10.1128/AAC.02643-15
Oguri, T., Furuyama, T., Okuno, T., Ishii, Y., Tateda, K., Bonomo, R. A., et al. (2014). Crystal structure of Mox-1, a unique plasmid-mediated Class C betaLactamase with hydrolytic activity towards moxalactam. Antimicrob. Agents Chemother. 58, 3914-3920. doi: 10.1128/AAC.02363-13

Oguri, T., Ishii, Y., and Shimizu-Ibuka, A. (2015). Conformational change observed in the active site of Class C beta-Lactamase MOX-1 upon binding to aztreonam. Antimicrob. Agents Chemother. 59, 5069-5072. doi: 10.1128/AAC.04428-14

Ouellette, M., Bissonnette, L., and Roy, P. H. (1987). Precise insertion of antibiotic resistance determinants into Tn21-like transposons: nucleotide sequence of the OXA-1 beta-lactamase gene. Proc. Natl. Acad. Sci. U.S.A. 84, 7378-7382.

Powers, R. A., Swanson, H. C., Taracila, M. A., Florek, N. W., Romagnoli, C., Caselli, E., et al. (2014). Biochemical and structural analysis of inhibitors targeting the ADC-7 cephalosporinase of Acinetobacter baumannii. Biochemistry 53, 7670-7679. doi: 10.1021/bi500887n

Vagin, A., and Teplyakov, A. (2010). Molecular replacement with MOLREP. Acta Crystallogr. D Biol. Crystallogr. 66, 22-25.

Wilke, M. S., Lovering, A. L., and Strynadka, N. C. (2005). $\beta$-Lactam antibiotic resistance: a current structural perspective. Curr. Opin. Microbiol. 8, 525-533. doi: 10.1016/j.mib.2005.08.016

Wu, G., Robertson, D. H., Brooks, C. L. III, and Vieth, M. (2003). Detailed analysis of grid-based molecular docking: a case study of CDOCKER-A CHARMmbased MD docking algorithm. J. Comput. Chem. 24, 1549-1562. doi: 10.1002/ jcc.10306

Author Disclaimer: The content is solely the responsibility of the authors and does not necessarily represent the official views of the NIH or the Department of Veterans Affairs.

Conflict of Interest: The authors declare that the research was conducted in the absence of any commercial or financial relationships that could be construed as a potential conflict of interest.

Publisher's Note: All claims expressed in this article are solely those of the authors and do not necessarily represent those of their affiliated organizations, or those of the publisher, the editors and the reviewers. Any product that may be evaluated in this article, or claim that may be made by its manufacturer, is not guaranteed or endorsed by the publisher.

Copyright (c) 2021 Ishikawa, Furukawa, Caselli, Prati, Taracila, Bethel, Ishii, Shimizu-Ibuka and Bonomo. This is an open-access article distributed under the terms of the Creative Commons Attribution License (CC BY). The use, distribution or reproduction in other forums is permitted, provided the original author(s) and the copyright owner(s) are credited and that the original publication in this journal is cited, in accordance with accepted academic practice. No use, distribution or reproduction is permitted which does not comply with these terms. 